\title{
Study of novel $d 1$ alleles, defective mutants of the $\alpha$ subunit of heterotrimeric G-protein in rice
}

\author{
Katsuyuki Oki ${ }^{1}$, Noriko Inaba ${ }^{1}$, Hidemi Kitano ${ }^{2}$, Sachiko Takahashi ${ }^{1}$, \\ Yukiko Fujisawa ${ }^{1}$, Hisaharu Kato ${ }^{1}$ and Yukimoto Iwasaki ${ }^{1 *}$ \\ ${ }^{1}$ Department of Bioscience, Fukui Prefectural University, 4-1-1 Matsuoka Kenjyojima, Eiheiji-cho, \\ Yoshida-gun, Fukui 910-1195, Japan \\ ${ }^{2}$ Bioscience and Biotechnology Center, Nagoya University, Chikusa, Nagoya 464-8604, Japan
}

(Received 4 December 2008, accepted 6 January 2009)

It has been shown that the disruption of the $\alpha$-subunit gene of heterotorimeric G-proteins $(\mathrm{G} \alpha)$ results in dwarf traits, the erection of leaves and the setting of small seeds in rice. These mutants are called $d 1$. We have studied the expression profiles of the transcripts and translation products of rice $\mathrm{G} \alpha$ in ten alleles of $d 1$ including five additional alleles newly identified. By RT-PCR, the transcripts

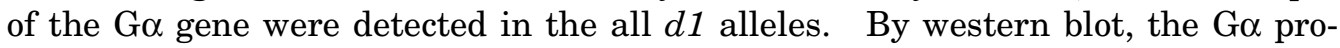
teins were not detected in the plasma membrane fractions of the $d 1$ alleles with the exception of d1-4. In $d 1-4$, one amino acid change in the GTP-binding box A of the $G \alpha$ protein was occurred and even in this case the $G \alpha$ protein was only just detectable in the plasma membrane fraction. Given that the G $\alpha$ protein did not accumulate in the plasma membrane fraction in $d 1-8$ which has a deletion of just a single amino acid in the G $\alpha$ protein, it is likely that a proper conformation of the $\mathrm{G} \alpha$ is necessary for accumulation of $\mathrm{G} \alpha$ protein in the plasma membrane. Nine alleles of $d 1$ showed a severer phenotype whilst $d 1-4$ exhibited a mild phenotype with respect to seed size and elongation pattern of internodes. As brassinosteroid signaling was known to be partially impaired in $d 1 \mathrm{~s}$, the sensitivity to 24epibrassinolide (24-epiBL) was compared among $d 1$ alleles in a T65 genetic background. Only $d 1-4$ showed responses similar to wild type rice. The results show that the $d 1-4$ mutant is a mild allele in terms of the phenotype and mild hyposensitivity to the exogenously applied 24-epiBL.

Key words: brassinosteroid, $d 1$ mutant, G-protein $\alpha$ subunit, mild-allele, rice

\section{INTRODUCTION}

Heterotrimeric G-proteins are signaling components regulating intracellular effector molecules after perception of signals by receptors in eukaryotes. They are composed of three subunits, namely $\mathrm{G} \alpha, \mathrm{G} \beta$, and $\mathrm{G} \gamma$. In mammals, signals transduced by these components are essential for a broad range of biological events, such as long term potentiation in neural communication (Goh and Pennefather, 1989) and immune responses (Ngai et al., 2008) as well as endocrine regulation (Landis et al., 1989). In rice plants, mutants of the Ga gene (RGA1), which is a single copy gene in the rice genome (Ishikawa et al., 1995), are called $d 1$ (Ashikari et al., 1999; Fujisawa et al., 1999). The $d 1$ mutants show abnormal gross morphologies, such as dwarfism, shortened internodes, small-rounded seeds, and erect leaves. They successfully

\section{Edited by Koji Murai}

* Corresponding author. E-mail: iwasaki@fpu.ac.jp reproduce the next generation and complete their life cycles. Thus, rice Go is not requisite for survival but is important for architecture of the body plan with a proper plant size. It is shown that the G $\alpha$ subunit is a determinant of the cell number in Arabidopsis (Ullah et al., 2001). However, the molecular mechanism via which the $\mathrm{G} \alpha$ promotes cell proliferation has not been fully elucidated as yet.

We analyzed sequences of RGA $1 \mathrm{cDNAs}$ in rice mutants displaying $d 1$-like phenotypes and obtained ten alleles of $d 1$. We also investigated whether mutated $\mathrm{G} \alpha$ are accumulated in plasma membrane fractions because rice $\mathrm{G} \alpha$ was shown to be localized in the plasma membrane (Kato et al., 2004). The results demonstrate that all of the $d 1$ alleles, except for $d 1-4$, may be considered to be null mutants, because the $G \alpha$ proteins were not detected in the plasma membrane fractions. As a result, we propose the possibility that the conformation of the rice G $\alpha$ may be strictly controlled, although a precise mechanism is not clear. 
A mild allele is considered to be useful for genetic and biochemical study. However, to our knowledge there is no report of a mild mutant of $\mathrm{G} \alpha$ in plants, including Arabidopsis and rice. In this study, we report one mild allele of $d 1$, the $d 1-4$ allele. This allele set longer seeds in the longitudinal direction and had longer second internodes than the other null alleles. Recent studies showed that Arabidopsis $\mathrm{G} \alpha$ partially participated in the brassinosteroid (BR) response (Ullah et al., 2002). In rice, $d 1$ had a decreased sensitivity to 24-epiBL, the most bioactive form of BR (Wang et al., 2006). The sensitivity to 24-epiBL in $d 1-4$ was similar to that of the wild type rice. These facts are in good accordance with the proposal that $d 1-4$ is a mild allele of $d 1$. We renamed the ten $d 1$ alleles as a series with the purpose of unifying the name of $d 1$ alleles and have summarized these mutation types including the expression pattern of mRNA, the accumulation profiles of the protein and phenotype of these alleles.

\section{MATERIALS AND METHODS}

Plant materials Four rice cultivars (Oryza sativa L. cv. Taichung 65, Nipponbare, Kinmaze and Blue Rose) and thirteen alleles of $d 1$ (T65d1, DKT1, DKT2, DKT3, DKT4, DKT5, TCM7-1198, DK22, CM232-2, CM1361-1, L32, B2X2-1 and B2X2-2, which are local names of strains) were used in this study. The DNA sequences of RGA1 in T65d1, DKT1, DKT2, DK22 and CM1361-1 were previously reported (Fujisawa et al., 1999). DKT3, DKT5, TCM7-1198, DKT4, CM232-2, L32, B2X2-1 and B2X2-2 were investigated as new alleles of $d 1$ in this study. DKT1, DKT3 and DKT5 had the same mutation in the G $\alpha$ gene. DKT2 and TCM7-1198 also had the same mutation in the $\mathrm{G} \alpha$ gene. The recurrent parent of T65d1, DKT1, DKT2, DKT3, DTK4, DKT5 and TCM71198 was Taichung 65. The recurrent parent of DK22 was Nipponbare. The recurrent parent of CM232-2 and CM1361-1 was Kinmaze. The recurrent parent of L32, B2X2-1 and B2X2-2 was Blue rose. We renamed T65d1 as $d 1-1$, DKT1 as $d 1-2$, DKT2 as $d 1-3$, DKT4 as $d 1-4$, DK22 as $d 1-5$, CM232-2 as $d 1-6$, CM1361-1 as $d 1-7$, L32 as $d 1-8, \mathrm{~B} 2 \mathrm{X} 2-1$ as $d 1-9$ and B2X2-2 as $d 1-10$. $d 2-1$, a BR-deficient mutant (Hong et al., 2003) and d61-2, a BRinsensitive mutant (Nakamura et al., 2006; Yamamuro et al., 2000) were used for the Lamina joint test.

\section{Growth conditions for morphological observation} After seeds were sown, plants were grown to the seedling stage in a green house. Subsequently, they were transferred to the paddy field and cultivated under sunlight.

Sequencing confirmation of the G $\alpha$ genes Total RNAs were extracted from the seedlings of the thirteen alleles of $d 1$ and four recurrent parents using the RNeasy
Mini Kit (Qiagen K. K., Japan). cDNAs were synthesized with the SuperScript III system (Invitrogen Japan K. K., Japan), using the total RNAs. DNA fragments including the coding region of $\mathrm{G} \alpha$ were amplified by PCR with the KOD-Plus system (TOYOBO Ltd., Japan). OsUbiquitin1 was used as an internal standard. Primers used for PCR were as follows: rice G $\alpha, R G A 1$ (forward: 5'- ctcaggcatgagagcatacc -3'/ reverse: 5'- ttgttcctctcgcegtcctaattac -3'), OsUbiquitin1 (forward: 5'-cacctgttctagggttcacaagtctgc-3'/ reverse: 5'-gcaaaatttggacacaatgattagggatc-3'). The coding region of $\mathrm{G} \alpha$ was sequenced by ABI PRISM 3100 Genetic Analyzer system.

Purification of the plasma membrane fractions and western blot analysis Plasma membrane (PM) fractions were prepared from seedlings grown in a growth chamber under $14 \mathrm{~h}$ light at $30^{\circ} \mathrm{C} / 10 \mathrm{~h}$ dark at $25^{\circ} \mathrm{C}$ conditions for 7 days. PM fractions were purified according to the previously described method (Kato et al., 2004). Briefly, the aerial parts were homogenized with four times volume of the grinding buffer. The homogenates were centrifuged at $10,000 \mathrm{~g}$ for $20 \mathrm{~min}$ and the supernatants were centrifuged at $100,000 \mathrm{~g}$ for $60 \mathrm{~min}$. The precipitates were further purified by an aqueous twophase partition method to obtain the PM fraction. The fractions were separated by SDS-PAGE using a 5-15\% gradient gel and blotted onto PVDF membrane. Antibody against recombinant rice $\mathrm{G} \alpha$ proteins was produced in a rabbit and used for western blotting as described previously (Kato et al., 2004).

Lamina joint inclination assay with 24-epiBL Seeds were surface-sterilized with $70 \% \mathrm{EtOH}$ for $30 \mathrm{sec}$ and then with $1.25 \%(\mathrm{w} / \mathrm{v})$ hypochlorous acid and $0.02 \%$ (w/v) Tween 20 for 15 min. After an imbibition at $22^{\circ} \mathrm{C}$ for 3 days and subsequently $30^{\circ} \mathrm{C}$ for 3 days, seeds were transferred to $0.6 \%$ agar medium and grown in an incubator room in continuous light at $30^{\circ} \mathrm{C}$ until the third leaves begin to emerge (4 days). Then, various amounts of 24-epiBL (a brassinolide analog, 24-epi-brassinolide; 635-00811, which was purchased from Wako Pure Chemical Industries, Ltd., Japan) were applied to the lamina regions between the second leaf blade and leaf sheath. After 3 days, the bending angles of lamina joint were measured.

\section{RESULTS}

Sites of the mutations in $\mathbf{G} \alpha$ genes of the ten $d 1$ alleles In order to find the mutation sites in the $d 1$ alleles, total RNAs derived from the $d 1$ alleles and their recurrent parents were isolated and the coding regions of G $\alpha$ were amplified by RT-PCR. Transcript abundances and size of $\mathrm{G} \alpha$ slightly varied among them (Fig. 1a). All fragments obtained by RT-PCR were sequenced and ten 
(a)

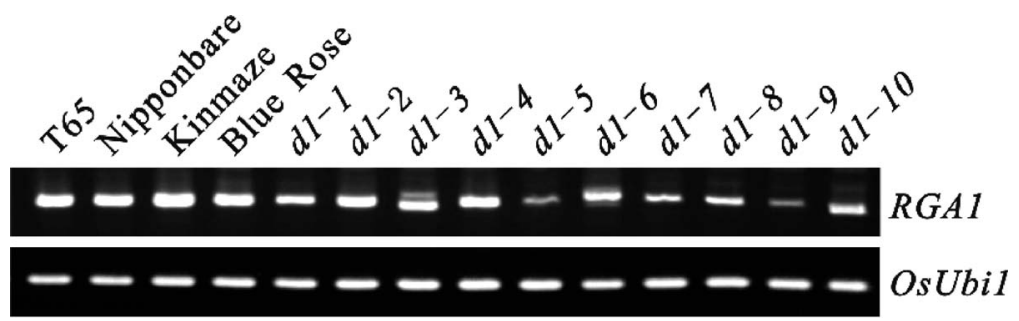

(b)

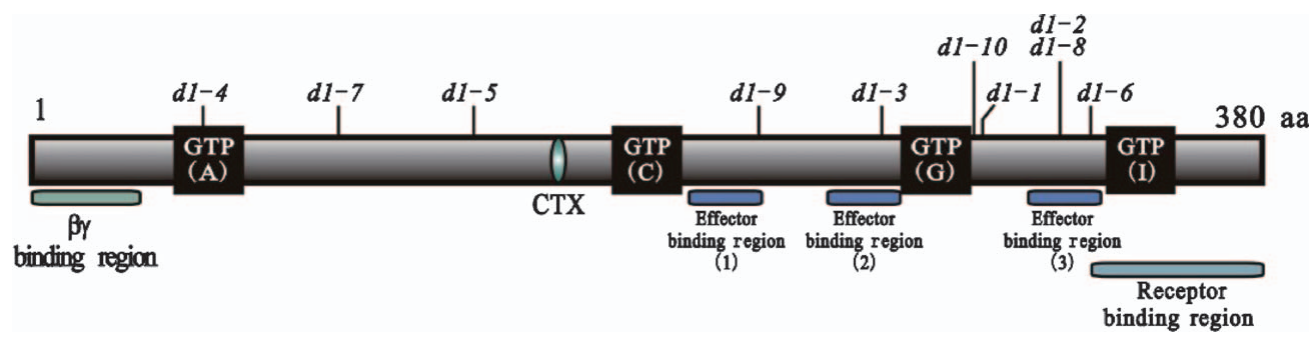

(c)

\begin{tabular}{clccccc}
\hline Allele & & Mutation & & Predicted size $(\mathrm{kDa})$ & Phenotype & Local name \\
\hline$d 1-1$ & 2 base deletion & $(\Delta 908-909)$ & preterm stop codon & 35.3 & severe & T65d1 (ID-1) \\
$d 1-2$ & 1 base substitution & $\left(\mathrm{A}^{985} \rightarrow \mathrm{T}^{985}\right)$ & preterm stop codon & 37.9 & severe & DKT1, DKT3, DKT5 \\
$d 1-3$ & 48 base deletion & $(\Delta 842-889)$ & 16 amino acid deletion & 42.2 & severe & DKT2, TCM7-1198 \\
$d 1-4$ & 1 base substitution & $\left(\mathrm{G}^{152} \rightarrow \mathrm{A}^{152}\right)$ & $\mathrm{G}^{51} \rightarrow \mathrm{E}^{51}$ & 44.3 & mild & DKT4 \\
$d 1-5$ & 1 base substitution & $\left(\mathrm{G}^{508} \rightarrow \mathrm{A}^{508}\right)$ & preterm stop codon & 19.1 & severe & DK22 \\
$d 1-6$ & 65 base insertion & $(977-(+65)-978)$ & preterm stop codon & 37.7 & severe & CM232-2 \\
$d 1-7$ & 19 base insertion & $(264-(+19)-265)$ & preterm stop codon & 10.7 & severe & CM1361-1 \\
$d 1-8$ & 3 base deletion & $(\Delta 991-993)$ & one amino acid deletion & 44.1 & severe & L32 \\
$d 1-9$ & 29 base deletion & $(\Delta 718-746)$ & preterm stop codon & 27.2 & severe & B2X2-1 \\
$d 1-10$ & 80 base deletion & $(\Delta 901-980)$ & preterm stop codon & 35.2 & severe & B2X2-2
\end{tabular}

Fig. 1. The mutation position of $d 1$ alleles. (a) RT-PCR of rice G $\alpha$ transcripts in four WT plants, namely T65, Nipponbare, Kinmaze and Blue Rose, and ten $d 1$ alleles including five newly identified $d 1$ alleles, namely $d 1-4, d 1-6, d 1-8, d 1-9$ and $d 1-10$. DNA fragments covering the entire coding region of $\mathrm{G} \alpha$ were amplified. OsUbi1 was used as an internal standard. (b) Schematic diagrams of the mutations in G $\alpha$ protein. (c) List of the type of mutations, predicted translational products, severity of the mutant phenotypes and names of $d 1$ alleles.

patterns of mutation sites were found (Fig. 1b). The explanation of mutation sites and the size of the predicted open reading frame are shown in Fig. 1c. To simplify the naming of these mutants we renamed T65d 1 as $d 1-1$, DKT1 as $d 1-2$, DKT2 as $d 1-3$, DKT4 as $d 1-4$, DK22 as d1-5, CM232-2 as d1-6, CM1361-1 as d1-7, L32 as d18 , B2X2-1 as d1-9 and B2X2-2 as d1-10. As five of the alleles $(d 1-4, d 1-6, d 1-8, d 1-9$ and $d 1-10)$ were new alleles, we describe the mutation sites in these alleles in this paper. The mutation sites in the other alleles of $d 1$ have been reported previously (Fujisawa et al., 1999). Here, the first base in ATG coding the first Met of RGA1 cDNA was designated as position 1 . A one base substitution from $\mathrm{G}$ to $\mathrm{A}$ at position 152 of RGA1 cDNA causes a one amino acid substitution from $\mathrm{Gly}^{51}$ to $\mathrm{Glu}^{51}$ in the GTP binding box A in d1-4. A one base substitution from $G$ to $A$ in the first base of thirteenth intron leads to a failure in a correct splicing at the junction between thirteenth exon and thirteenth intron in $d 1$ 6. This leads to the insertion of an additional 65 bases derived from the remnant of thirteenth intron in the site between position 977 and 978 of RGA1 cDNA and causes a premature stop codon in d1-6. The deletion of 3 bases between positions 991 and 993 of RGA1 cDNA causes the deletion of one amino acid $\left(\mathrm{K}^{331}\right)$ in $d 1-8$. The deletion of 29 bases between positions 718 and 746 of RGA1 cDNA introduces a premature stop codon in $d 1-9$. The deletion of 80 bases between positions 901 and 980 of RGA 1 cDNA produces a premature stop codon in $d 1-10$. Among the ten alleles, the $d 1-3, d 1-4$ and $d 1-8$ mutants were inframe mutants, resulting in a 16 amino acid deletion, a one amino acid exchange and a one amino acid deletion, respectively.

Protein levels of $\mathrm{G} \alpha$ in the plasma membrane fractions of $d 1$ alleles Plant Gos of heterotrimeric G proteins are considered to be membrane-anchored proteins with postulated myristylation and palmitoylation moieties in their N-termini (Adjobo-Hermans et al., 2006). The rice G $\alpha$ protein, which consists of 380 amino acid residues and has a theoretical molecular weight of 44.2 $\mathrm{kDa}$, was shown to be localized in a plasma membrane 
fraction as a $46 \mathrm{kDa}$ species by SDS-PAGE (Kato et al., 2004). We performed western blot analysis with the plasma membrane fractions of the ten $d 1$ alleles using a polyclonal antibody raised against $\mathrm{G} \alpha$ (Fig. 2). As a result, no $\mathrm{G} \alpha$ could be detected in the plasma membrane with the exception of $d 1-4$, for which faint protein band were observed as indicated by an arrow head (Fig. 2). As previously mentioned, three alleles, namely $d 1-3, d 1-4$ and $d 1-8$, are in-frame mutants, for which the predicted open reading frames are 364,380 and 379 amino acids, respectively. The size of the mutated $\mathrm{G} \alpha$ proteins was expected to be about $42.2 \mathrm{kDa}$ in $d 1-3,44.3 \mathrm{kDa}$ in $d 1-4$ and $44.1 \mathrm{kDa}$ in $d 1-8$. G $\alpha$ protein was not accumulated in $d 1-8$ and was barely present in $d 1-4$. It was not clear whether the G $\alpha$ proteins accumulated in the case of $d 1-3$ because of the presence of non-specific bands at the expected size of the mutated $\mathrm{G} \alpha(42.2 \mathrm{kDa})$.

\section{Gross and seed morphologies of the ten $d 1$ alleles}

The $d 1$ mutants reported above showed plural characteristic phenotypes (Ashikari et al., 1999; Fujisawa et al., 1999). They showed erect and shortened leaves with a dark green color. The elongation of internodes is inhibited and showed dwarfism in $d 1 \mathrm{~s}$. Seeds are specifically shortened in the vertical direction in $d 1 \mathrm{~s}$. The gross morphology at the grain-filling stage and seed morphologies of the ten $d 1$ alleles are shown in Fig. $3 \mathrm{a}$ and $3 \mathrm{~b}$, respectively. The seed size in the vertical and horizontal axis and seed weight were measured (Fig. 4). In addition, lengths of the longest 12 internodes of plants were measured and expressed as a relative value (Fig. 5a and $5 b)$. The numbers of the tillers with $\mathrm{dm}$ or $\mathrm{d} 6$ type among 12 tillers in a plant are also shown in Fig. 5c. All $d 1$ alleles possess these $d 1$ characteristics (Figs. 3, 4 and 5).

Among them, d1-4 showed mild phenotypes, compared with $d 1-1, d 1-2$ and $d 1-3$ of which the recurrent parent is Taichung 65 (T65). The $\mathrm{dm}$ or $\mathrm{d} 6$ elongation patterns (Takeda, 1977) are a hallmark of a BR-deficient phenotype, such as is seen for $d 61-2$ (Yamamuro et al., 2000). It was also observed in $d 1-1, d 1-2$ and $d 1-3$, in which the recurrent parent was T65. However, the $d 1-4$ mild allele with the T65 genetic background scarcely demonstrated such a dm phenotype. Seeds of $d 1-4$ were bigger than $d 1-1, d 1-2$ and $d 1-3$. These properties support the proposition that $d 1-4$ is a mild allele of $d 1$. In contrast, the novel $d 1-6$ allele exhibited a somewhat more severe phenotype than $d 1-7$ under the same genetic background, namely Kinmaze. In the western blot analysis of these alleles, both seemed to be null. We can not find a reasonable mechanism to explain the differences in phenotypes between $d 1-6$ and d1-7. The novel alleles, $d 1-8, d 1-9$, and $d 1-10$ showed dwarfism and set small seeds, compared with Blue Rose, the recurrent parent. They may be considered to be null alleles, by western blot analysis.

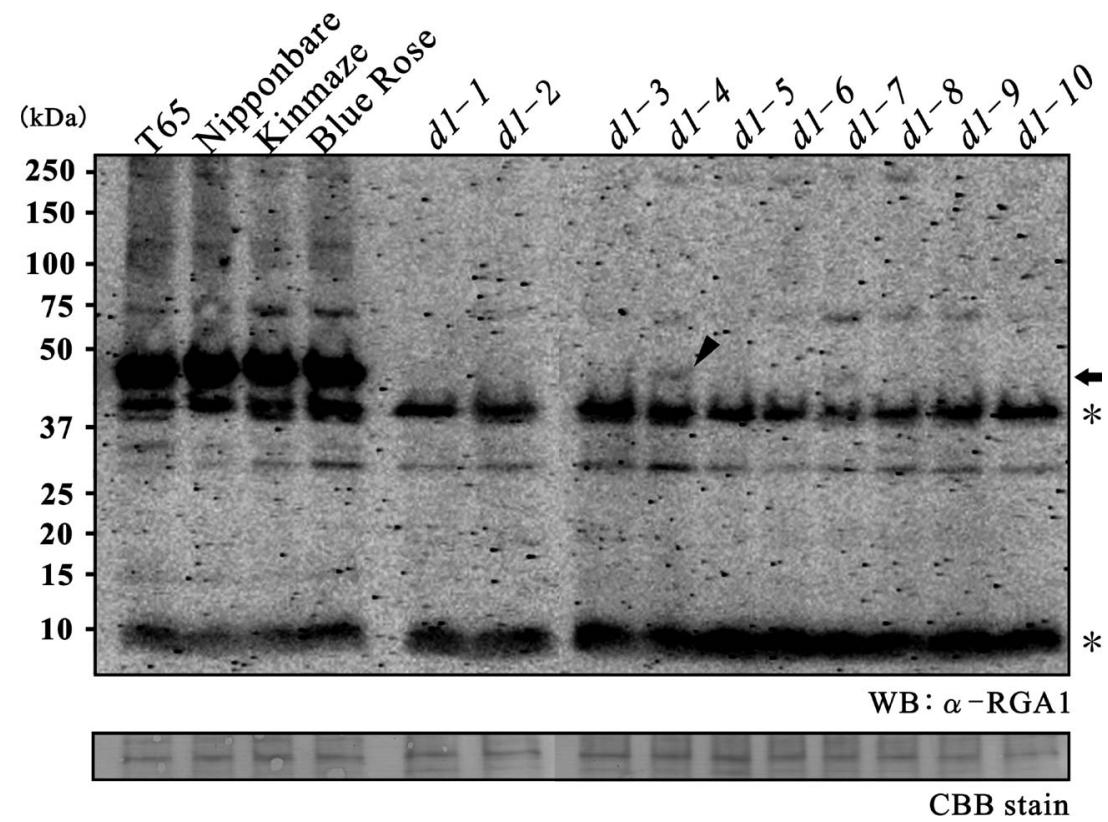

Fig. 2. Analysis of the mutated G $\alpha$ proteins in $\mathrm{dl}$ alleles. Western blot analysis of plasma membrane fractions of the ten $d 1$ alleles and four WT rice cultivars. G $\alpha$ proteins were detected as bands of approximately $46 \mathrm{kDa}$ by Western blot analysis using an anti-G $\alpha$ polyclonal antibody in four WT plants (arrow). No G $\alpha$ could be detected in any of the $d 1$ alleles except for $d 1-4$, in which faint protein bands were observed as indicated by an arrow head. Asterisks denote the non-specifically detected bands. 
Responses to 24-epiBL of the $d 1$ alleles with T65 genetic background Heterotrimeric G-proteins are involved in many plant hormone responses, such as those to abscisic acid (Pandey et al., 2006), gibberellic acid (GA) (Chen et al., 2004) and ethylene (Ullah et al., 2002). Previously, Go mutants were showed to be hyposensitive to exogenously applied 24-epiBL, one of the bioactive forms of $\mathrm{BR}$, in rice (Wang et al., 2006) and Arabidopsis (Chen et al., 2004; Ullah et al., 2002). We tested whether $d 1-4$ showing a mild phenotype exhibited a mild response to 24 -epiBL by lamina joint inclination assay (Fig. 6). The four $d 1$ alleles, $d 1-1, d 1-2, d 1-3$ and d1-4 together with $d 61-2$ which is a mutant of the BR receptor gene (OsBRI1) and $d 2-1$ which is a mutant of a

(a)

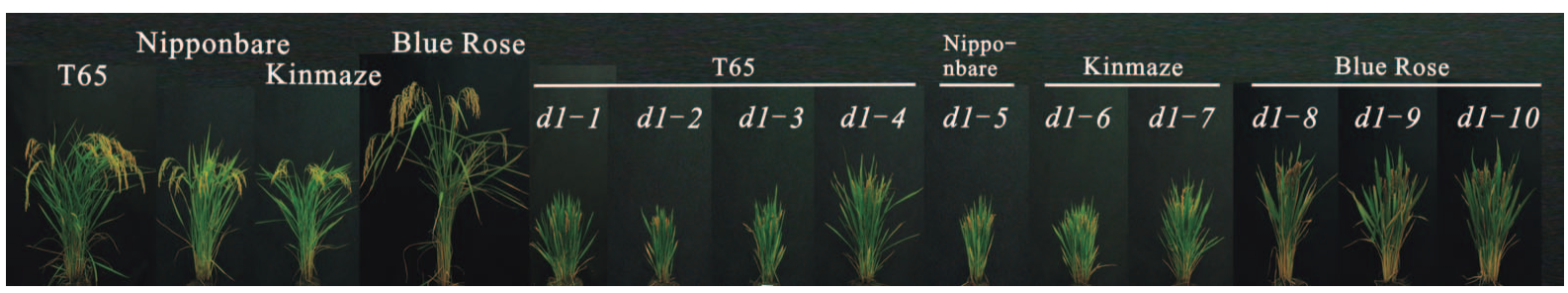

(b)

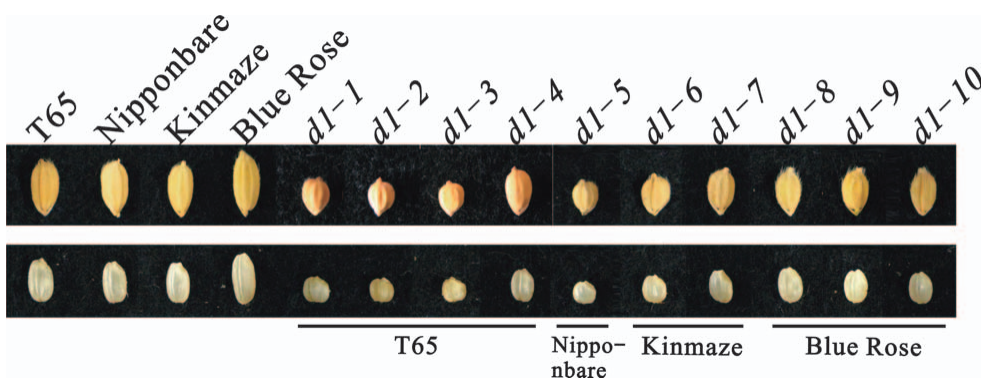

Fig. 3. Morphological comparison among $d 1$ alleles. (a) Gross morphologies of the four WT plants and ten $d 1$ alleles at the grainfiling stage. The recurrent parents of each $d 1$ allele are depicted above them. (b) Seed morphologies of the plants in (a). The upper panel and the lower panel represent the unhulled and hulled seeds respectively. The names of recurrent parents of each $d 1$ allele are shown below them.
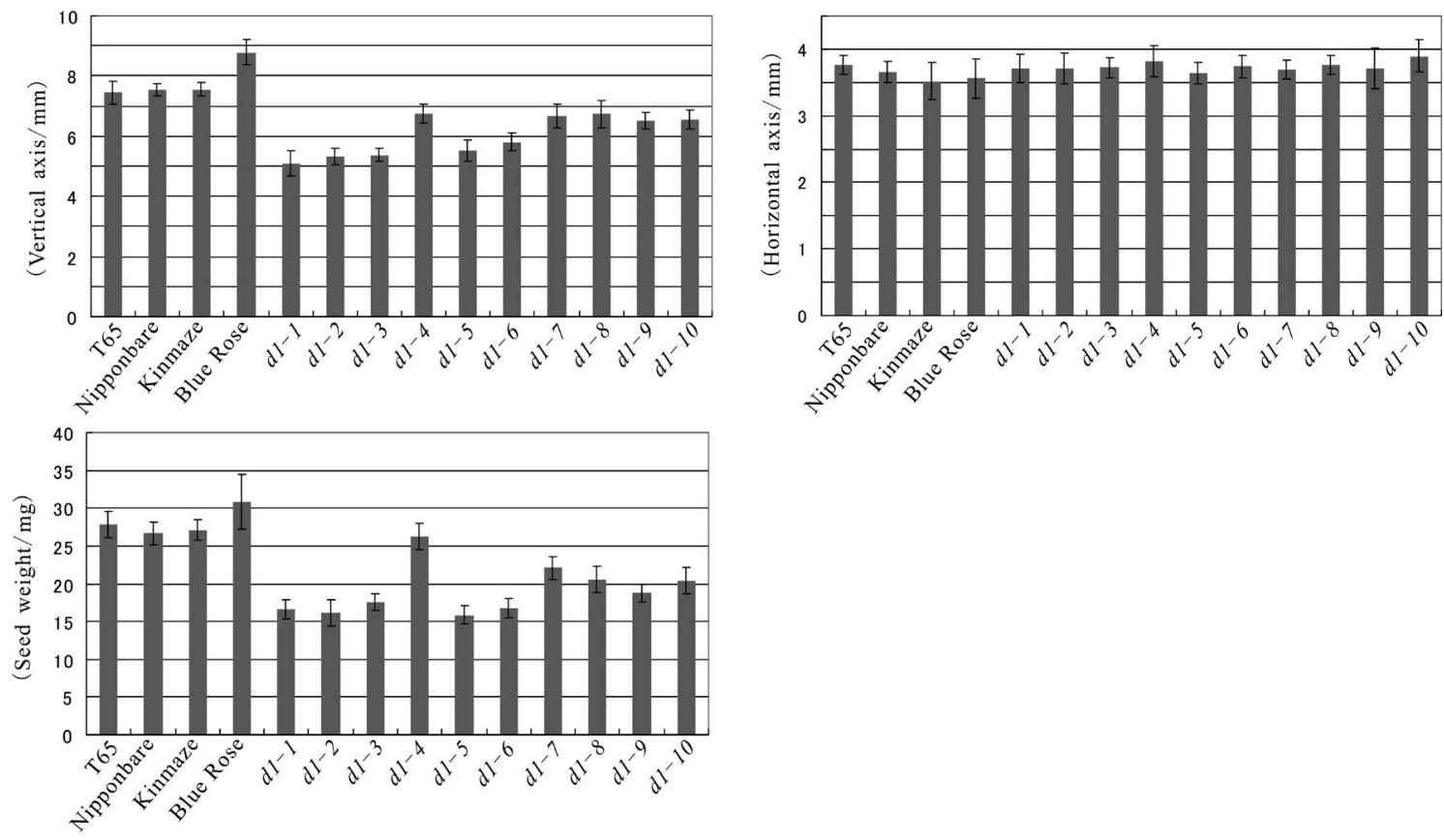

Fig. 4. Measurement of the seed size and weight of $d 1$ alleles. The seed lengths in vertical and horizontal axis were measured for the four WT plants and ten $d 1$ alleles (upper two graphs). Seed weights were also measured (lower graph). Data is the average of ten seeds (Error bar, SD). 
(a)

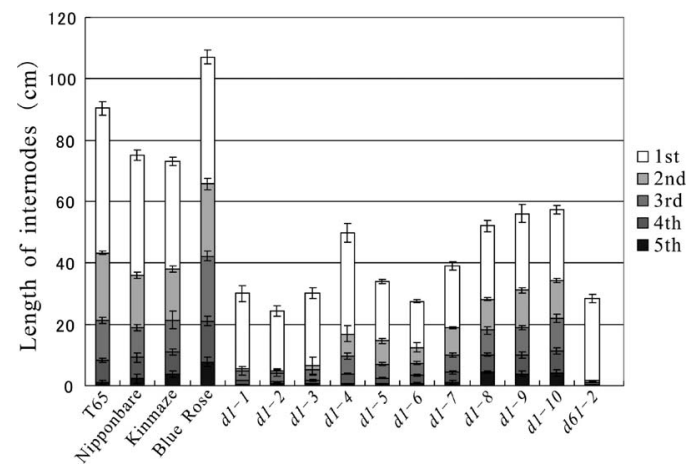

(c)

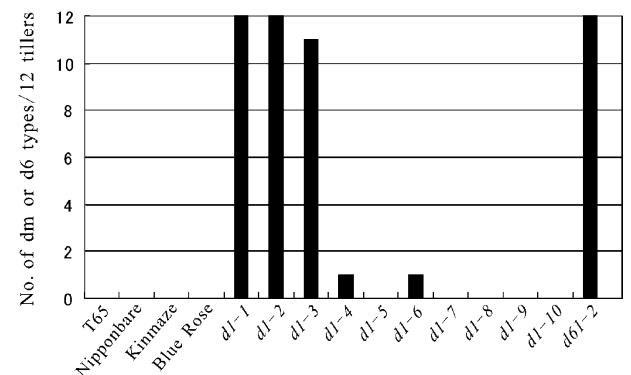

(b)

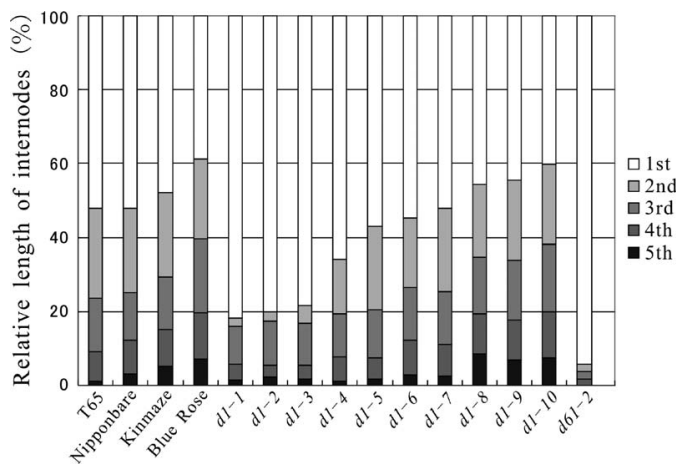

Fig. 5. Measurement of the internodes lengths of $d 1$ alleles. The lengths from first to fifth internodes for four WT plants and ten $d 1$ alleles, as well as OsBRI1 mutant and d61-2 were measured. Shown are the actual values (a), the relative length of internodes (b), and the frequency of a dm or d6 type tiller per 12 tillers in a plant (c).
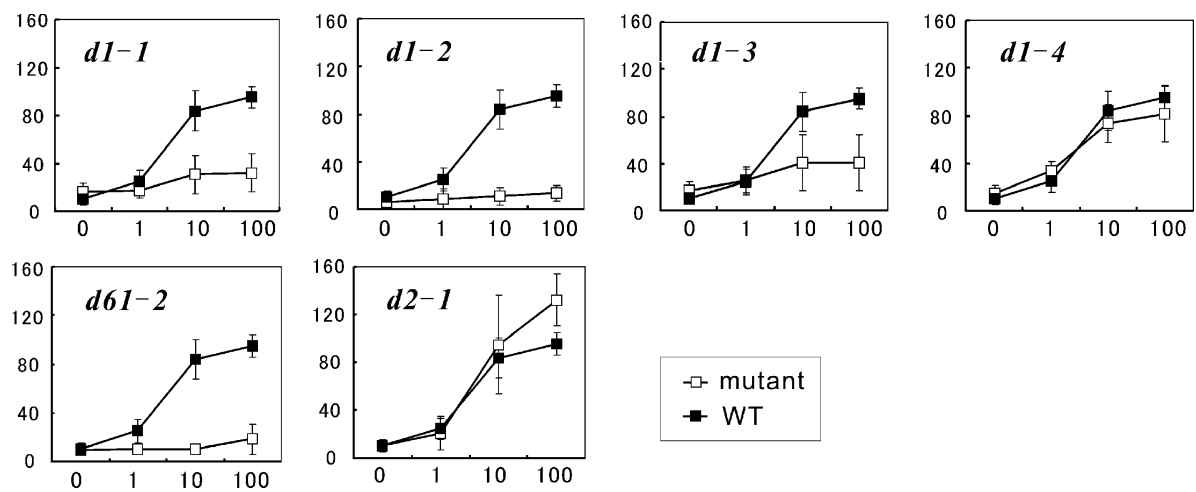

$\rightarrow$ WT

Fig. 6. Lamina joint inclination assay of $d 1$ alleles. Comparison of the sensitivity of the four $d 1$ alleles as well as $d 61-2, d 2-1$ to exogenously applied 24-epiBL. The recurrent parent of all these plants is T65. The $d 61-2$ and $d 2-1$ were used as typical BR-hyposentitive and hypersensitive mutants respectively. The black squares and white squares are the bending angle of the lamina part in WT (T65) and each mutant. The yaxis is the bending angle $\left(^{\circ}\right)$ and $x$-axis is the amount of 24-epiBL inoculated onto the lamina joint region (ng). The values are the average of 18 samples (Error bar, SD).

gene coding a BR biosynthetic enzyme (CYP90D) were derived from the same genetic background, T65. As it has already been shown that unexpected differences were caused by having a different parent in Arabidopsis, even in mutation of the same gene (Trusov et al., 2008), we paid close attention and chose mutants with the same genetic background. First, we confirmed that our experimental conditions were suitable using $d 61-2$ and $d 2-1$, which are known to show an insensitivity and hyper sensitivity, respectively. The results with the four $d 1$ alleles showed a decreased bending angle compared to that of wild type (WT). d1-1, d1-2, and d1-3 mutants showed the least sensitivity to 24-epiBL. The angle of $d 1-1, d 1$ 2, d1-3 mutants and WT at $100 \mathrm{ng}$ of 24-epiBL were $32.1^{\circ}$, $13.6^{\circ}, 40.4^{\circ}$ and $95.1^{\circ}$ respectively. In contrast to $d 1-1$, $d 1-2$ and $d 1-3$, the bending angle of the $d 1-4$ at $100 \mathrm{ng}$ of 24-epiBL was $81.7^{\circ}$ which is close to the WT level. The results show that $d 1-4$ has a mildly impaired sensitivity to exogenously applied 24-epiBL. 


\section{DISCUSSION}

The heterotrimeric G-proteins are pivotal signaling elements and are a multiple gene family in metazoans (McCudden et al., 2005). In contrast to metazoans, restricted numbers of gene are known in plants. Indeed, there is only one canonical $\alpha$ subunit gene, one canonical $\beta$ subunit gene, one canonical and one atypical $\gamma$ subunit gene with an extra-stretch of amino acid in the $\mathrm{N}$ terminus thus far known in plants studied to date (Kato et al., 2004). Despite the simplified repertoire of $\mathrm{G} \alpha$ in plants, plant Gos are concerned with signaling of many plant hormones (Chen et al., 2004; Pandey et al., 2006; Ullah et al., 2002), blue light (Warpeha et al., 2007), and D-glucose (Huang et al., 2006). In rice plants, the mutants of a $\mathrm{G} \alpha$ gene, $d 1 \mathrm{~s}$ were originally identified in 1999 (Ashikari et al., 1999) and they have been used in subsequent G-protein studies (Komatsu et al., 2005; Ueguchi-Tanaka et al., 2000). $\quad d 1$ s exhibit specific multiple abnormities in the gross phenotype (Fig. 3).

Through the analysis of ten alleles of $d 1$, we have found some important points. First, we identified two in-frame mutants, $d 1-4$ and $d 1-8$, in addition to $d 1-3$ which had been previously reported (Fujisawa et al., 1999). Interestingly, the translation products in these three alleles of $d 1$ do not accumulate in (or do so at very low levels) the plasma membrane fraction unlike as is the case for WT. This result suggests that rice G $\alpha$ may be strictly regulated by a quality-control system concerned with protein conformation, though its mechanism is not currently clear. Study of post translational events may be more important in order to evaluate the stability of the $\mathrm{G} \alpha$ in rice.

Secondly, we have identified the first mild allele of $d 1$, d1-4. The internodes lengths and seed size in $d 1-4$ were longer and bigger than $d 1-1, d 1-2$ and $d 1-3$ (Figs. 3, 4 and 5 ), as well as having a greater bending angle of lamina joint region than $d 1-1, d 1-2$ and $d 1-3$ upon treatment with 24-epiBL (Fig. 6). Thus, $d 1-4$ showed mild phenotypes in plant height and seed size and exhibited a mildly altered response to exogenous 24-epiBL. The mutated G $\alpha$ protein in d1-4 may lose proper guanine-nucleotide binding or hydrolysis activity, because a single amino acid exchange was generated in GTP binding box A, which is highly conserved among Gos throughout eukaryotes. In the future, it will be important to clarify the enzymatic characteristics using recombinant protein having this mutation. If this mutation shows a GDP-binding form due to probable altered guanine-nucleotide binding properties, the recombinant protein may be useful as a bait in the isolation of a novel $\mathrm{G} \alpha$-binding protein which interacts with a $\mathrm{G} \alpha$ of the GDP-binding form.

We thank Dr. Tadashi Asahi for critical review of this manuscript. We thank Dr. Hikaru Satoh, Yasuo Nagato and
Kazuyoshi Takeda for the gift of $d 1$ alleles. This work was supported by grants from the Ministry of Agriculture, Forest and Fisheries of Japan (Project for Molecular Cloning and Characterization of Agronomically Important Genes, IPG0002) for Y. Iwasaki. It was further supported in part by Grants-in Aids for Scientific Research on Priority Areas (No.20061026) for Y. Iwasaki and in part by Grants-in Aids for JSPS Fellows (No. 18.2073) for K. Oki from the Ministry of Education, Culture, Sports, Science and Technology of Japan.

\section{REFERENCES}

Adjobo-Hermans, M. J., Goedhart, J., and Gadella, T. W., Jr. (2006) Plant G protein heterotrimers require dual lipidation motifs of $\mathrm{G} \alpha$ and $\mathrm{G} \gamma$ and do not dissociate upon activation. J. Cell Sci. 119, 5087-5097.

Ashikari, M., Wu, J., Yano, M., Sasaki, T., and Yoshimura, A. (1999) Rice gibberellin-insensitive dwarf mutant gene Dwarf 1 encodes the $\alpha$-subunit of GTP-binding protein. Proc. Natl. Acad. Sci. USA 96, 10284-10289.

Chen, J. G., Pandey, S., Huang, J., Alonso, J. M., Ecker, J. R., Assmann, S. M., and Jones, A. M. (2004) GCR1 can act independently of heterotrimeric G-protein in response to brassinosteroids and gibberellins in Arabidopsis seed germination. Plant Physiol. 135, 907-915.

Fujisawa, Y., Kato, T., Ohki, S., Ishikawa, A., Kitano, H., Sasaki, T., Asahi, T., and Iwasaki, Y. (1999) Suppression of the heterotrimeric $\mathrm{G}$ protein causes abnormal morphology, including dwarfism, in rice. Proc. Natl. Acad. Sci. USA 96, 7575-7580.

Goh, J. W., and Pennefather, P. S. (1989) A pertussis toxin-sensitive $\mathrm{G}$ protein in hippocampal long-term potentiation. Science 244, 980-983.

Hong, Z., Ueguchi-Tanaka, M., Umemura, K., Uozu, S., Fujioka, S., Takatsuto, S., Yoshida, S., Ashikari, M., Kitano, H., and Matsuoka, M. (2003) A rice brassinosteroid-deficient mutant, ebisu dwarf (d2), is caused by a loss of function of a new member of cytochrome P450. Plant Cell 15, 29002910.

Huang, J., Taylor, J. P., Chen, J. G., Uhrig, J. F., Schnell, D. J., Nakagawa, T., Korth, K. L., and Jones, A. M. (2006) The plastid protein THYLAKOID FORMATION1 and the plasma membrane G-protein GPA1 interact in a novel sugar-signaling mechanism in Arabidopsis. Plant Cell 18, 1226-1238.

Ishikawa, A., Tsubouchi, H., Iwasaki, Y., and Asahi, T. (1995). Molecular cloning and characterization of a cDNA for the $\alpha$ subunit of a G protein from rice. Plant Cell Physiol. 36, 353-359.

Kato, C., Mizutani, T., Tamaki, H., Kumagai, H., Kamiya, T., Hirobe, A., Fujisawa, Y., Kato, H., and Iwasaki, Y. (2004) Characterization of heterotrimeric G protein complexes in rice plasma membrane. Plant J. 38, 320-331.

Komatsu, S., Abbasi, F., Kobori, E., Fujisawa, Y., Kato, H., and Iwasaki, Y. (2005) Proteomic analysis of rice embryo: an approach for investigating $\mathrm{G} \alpha$ protein-regulated proteins. Proteomics 5, 3932-3941.

Landis, C. A., Masters, S. B., Spada, A., Pace, A. M., Bourne, H. R., and Vallar, L. (1989) GTPase inhibiting mutations activate the $\alpha$ chain of Gs and stimulate adenylyl cyclase in human pituitary tumours. Nature 340, 692-696.

McCudden, C. R., Hains, M. D., Kimple, R. J., Siderovski, D. P., and Willard, F. S. (2005) G-protein signaling: back to the future. Cell Mol. Life Sci. 62, 551-577.

Nakamura, A., Fujioka, S., Sunohara, H., Kamiya, N., Hong, Z., 
Inukai, Y., Miura, K., Takatsuto, S., Yoshida, S., UeguchiTanaka, M., et al. (2006) The role of OsBRI1 and its homologous genes, OsBRL1 and OsBRL3, in rice. Plant Physiol. 140, 580-590.

Ngai, J., Methi, T., Andressen, K. W., Levy, F. O., Torgersen, K. M., Vang, T., Wettschureck, N., and Tasken, K. (2008) The heterotrimeric G-protein $\alpha$-subunit Goq regulates TCRmediated immune responses through an Lck-dependent pathway. Eur. J. Immunol. 38, 3208-3218.

Pandey, S., Chen, J. G., Jones, A. M., and Assmann, S. M. (2006) G-protein complex mutants are hypersensitive to abscisic acid regulation of germination and postgermination development. Plant Physiol. 141, 243-256.

Takeda, K. (1977) Internode elongation and dwarfism in some gramineous plants. Gamma Field Symp. 16, 1-18.

Trusov, Y., Zhang, W., Assmann, S. M., and Botella, J. R. (2008) $\mathrm{G} \gamma 1+\mathrm{G} \gamma 2$ not equal to $\mathrm{G} \beta$ : heterotrimeric G protein $\mathrm{G} \gamma$-deficient mutants do not recapitulate all phenotypes of G $\beta$-deficient mutants. Plant Physiol. 147, 636-649.

Ueguchi-Tanaka, M., Fujisawa, Y., Kobayashi, M., Ashikari, M., Iwasaki, Y., Kitano, H., and Matsuoka, M. (2000) Rice dwarf mutant $d 1$, which is defective in the $\alpha$ subunit of the het- erotrimeric G protein, affects gibberellin signal transduction. Proc. Natl. Acad. Sci. USA 97, 11638-11643.

Ullah, H., Chen, J. G., Wang, S., and Jones, A. M. (2002) Role of a heterotrimeric $\mathrm{G}$ protein in regulation of Arabidopsis seed germination. Plant Physiol. 129, 897-907.

Ullah, H., Chen, J. G., Young, J. C., Im, K. H., Sussman, M. R., and Jones, A. M. (2001) Modulation of cell proliferation by heterotrimeric G protein in Arabidopsis. Science $\mathbf{2 9 2}$, 2066-2069.

Wang, L., Xu, Y. Y., Ma, Q. B., Li, D., Xu, Z. H., and Chong, K. (2006) Heterotrimeric G protein $\alpha$ subunit is involved in rice brassinosteroid response. Cell Res. 16, 916-922.

Warpeha, K. M., Upadhyay, S., Yeh, J., Adamiak, J., Hawkins, S. I., Lapik, Y. R., Anderson, M. B., and Kaufman, L. S. (2007) The GCR1, GPA1, PRN1, NF-Y signal chain mediates both blue light and abscisic acid responses in Arabidopsis. Plant Physiol. 143, 1590-1600.

Yamamuro, C., Ihara, Y., Wu, X., Noguchi, T., Fujioka, S., Takatsuto, S., Ashikari, M., Kitano, H., and Matsuoka, M. (2000) Loss of function of a rice brassinosteroid insensitive1 homolog prevents internode elongation and bending of the lamina joint. Plant Cell 12, 1591-1606. 